\title{
Data Repository Item 2006071
}

\section{Finite Element Modeling Methods}

The personally-developed finite element code follows standard methods as outlined in, for example, Smith and Griffiths (1998). Quadratic interpolation is used with 9-node quadrilateral elements for the models of Figs. 1 and 3, and 7-node triangles for the models of Fig. 2. In all cases pressure interpolation within the elements is linear, i.e. with three degrees of freedom, to avoid potential over-constraint and development of checkerboard patterns (Hughes, 2000). Pressure is discontinuous between elements and is eliminated at the element level before assembly of the global matrix. Symmetry of the global matrix is always maintained, in nonlinear cases (power law viscosity and plasticity) by placing non-linear terms on the RHS and iterating, and fast and efficient solution can therefore be achieved by Cholesky factorization. For Figs. 1 and 2, incompressible viscous rheology is employed, and solution is achieved using an iterative penalty function method to maintain the incompressibility requirement (Cuvelier et al., 1986, p. 274). Power-law viscous rheology with bell-shape strain softening is modeled in terms of an effective viscosity given by

$$
\eta_{e f f}=\frac{\eta \dot{\varepsilon}_{e f f}\left(\frac{1}{n}-1\right)}{1+\left(\varepsilon_{e f f} / \varepsilon_{r e f}\right)^{m}}
$$

for an effective strain rate $\dot{\varepsilon}_{\text {eff }}$ in plane strain given by

$$
\dot{\varepsilon}_{e f f}=\sqrt{\frac{1}{4}\left(\dot{\varepsilon}_{x x}-\dot{\varepsilon}_{y y}\right)^{2}+\dot{\varepsilon}_{x y}^{2}},
$$

where $\dot{\varepsilon}_{x x}, \dot{\varepsilon}_{y y}$, and $\dot{\varepsilon}_{x y}$ are the components of the rate-of-deformation tensor. In Eq. (1), $\eta$ is the viscosity at an effective strain rate of 1 and $n$ is the power law stress exponent. The strain softening exponent $m$ is equal to 2 in all models of Fig. 1. The effective viscous strain $\varepsilon_{\text {eff }}$ is obtained by summing, for each time increment, the appropriate effective strain increment $d \varepsilon$, which is given by (Malvern, 1969, p. 364)

$$
d \varepsilon=\sqrt{\frac{2}{9}\left[\left(d \varepsilon_{x x}-d \varepsilon_{y y}\right)^{2}+d \varepsilon_{x x}{ }^{2}+d \varepsilon_{y y}{ }^{2}\right]+\frac{1}{3} d \varepsilon_{x y}{ }^{2}} .
$$

In Fig. 1, the reference strain $\varepsilon_{r e f}$ is $10^{-2}$ in the strain softening experiments (2) and (3) and $10^{10}$ for experiment (1), without strain softening. In the models of Fig. 2, there is no strain softening and the denominator of Eq. (1) is always unity.

The full models of Fig. 1 have a length to width ratio of 4:1 and consist of $256 \mathrm{x}$ 32 quadrilateral elements. An element with $10 \%$ lower viscosity is introduced 7 elements left of the centre line and 15 elements from the base, as an initial perturbation to initiate localization. Velocity boundary conditions consistent with pure shear are imposed, with both upper and lower surfaces free and the left and right sides converging with an $\mathrm{x}$ velocity prescribed for constant strain rate. The $y$-velocity is unconstrained, except for the 
midpoint of the left side, where the y-velocity is fixed at zero. In Fig. 3 only the central third of the whole model is shown (i.e. ca. 85 x 32 elements).

The models of Fig. 2 have dimensions of 40 x 40 units. The viscosity of the material within the central ellipse is 10 times less than the matrix, the length to width ratio is 50 , the long axis is 4 units long and this axis is oriented at $60^{\circ}$ to the horizontal shortening direction. Triangular grids are constructed using the inbuilt routines of the PDE Toolbox of Matlab®, with 201 nodes around the perimeter of the ellipse. Models are presented after the very first increment of deformation in pure shear, with left and right $\mathrm{x}$-velocities prescribed, the central point of the model fixed, and all other velocities at the boundaries unconstrained.

The code for the model of Fig. 3 is for a linear elastic - linear viscous - Mohr Coulomb plastic material, but the viscosity is set sufficiently high that the material is effectively elastic-plastic. Implementation follows the methods outlined in Vermeer and de Borst (1984) and Smith and Griffiths (1998). For numeric stability, calculations are done in non-dimensional form, but the parameters scale to a strain rate of $10^{-14} \mathrm{~s}^{-1}$, an elastic shear modulus of $10^{10} \mathrm{~Pa}$, Poisson's ratio of 0.3 , viscosity of $10^{26} \mathrm{~Pa}$ s, angle of internal friction $30^{\circ}$, and dilatancy angle $0^{\circ}$. Cohesion is generally $10^{6} \mathrm{~Pa}$, but in one element it is $1 \%$ less to provide a locus for plastic yield. A background pressure of $10^{8} \mathrm{~Pa}$ (100 MPa) is imposed. The full model of Fig. 3 has a length to width ratio of 8:1, and consists of 512 x 64 quadrilateral elements. The element with slightly lower cohesion is immediately left of the centre line and third from the base. Velocity boundary conditions consistent with bulk pure shear are imposed, with the upper surface free, the base with yvelocity zero and $\mathrm{x}$-velocity unconstrained, the left and right sides converging with an $\mathrm{x}$ velocity prescribed for constant strain rate, and the $y$-velocity on these sides unconstrained. In Fig. 3 only the central third of the whole model is shown (i.e. ca. $170 \mathrm{x}$ 64 elements).

\section{References}

Cuvelier, C., Segal, A., and van Steenhoven, A.A., 1986, Finite Element Methods and Navier-Stokes Equations, D. Reidel, Dordrecht, 483 p.

Hughes, T.J.R, 2000, The Finite Element Method, Linear Static and Dynamic Finite Element Analysis, Dover Publications, 682 p.

Malvern, L.E., 1969, Introduction to the Mechanics of a Continuous Medium, PrenticeHall, 713 p.

Smith, I.M., and Griffiths, D.V., 1998, Programming the Finite Element Method ( ${ }^{\text {rd }}$ Ed), John Wiley \& Sons, 534 p.

Vermeer, P.A., and de Borst, R., 1984, Non-associated plasticity for soils, concrete and rock: Heron, v. 29, p. 1-64. 